\title{
Twist Contributes to Proliferation and Epithelial-to-Mesenchymal Transition-Induced Fibrosis by Regulating YB-1 in Human Peritoneal Mesothelial Cells
}

\author{
Lijie He, ${ }^{* \dagger}$ Mingwen Che, ${ }^{* \dagger}$ Jinping Hu, ${ }^{* \dagger}$ Sutong Li, ${ }^{* \S}$ Zhen Jia, ${ }^{*}$ Weijuan Lou, ${ }^{*}$ Cuixiang Li, ${ }^{*}$ Jun Yang, ${ }^{*}$ Shiren Sun, ${ }^{*}$ \\ Hanmin Wang, ${ }^{*}$ and Xiangmei Chen ${ }^{*}$
}

From the Department of Nephrology* and the State Key Laboratory of Cancer Biology, ${ }^{\dagger}$ Xijing Hospital, the Fourth Military Medical University, Xi'an, Shaanxi; the Department of Medicine, ${ }^{\ddagger}$ No. 273 Hospital of PLA, Korla, Xinjiang; the Department of Nephrology, ${ }^{\S}$ the Central Hospital of Xi'an, Xi'an, Shaanxi; the Department of Nephrology, "the First Hospital of Xi'an, Xi'an, Shaanxi; and the Department of Nephrology," State Key Laboratory of Kidney Diseases, Chinese PLA General Hospital and Medical College, Beijing, People's Republic of China

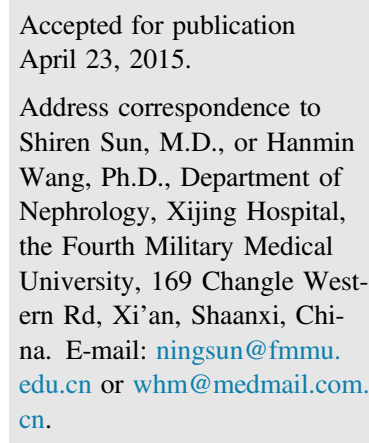

\begin{abstract}
Twist is overexpressed in high glucose (HG) damage of human peritoneal mesothelial cells (HPMCs) in vitro. Herein, we further identified its precise function related to fibrosis of peritoneal membranes (PMs). The overexpression and activation of Twist and YB-1 (official name, YBX1) and a transformed fibroblastic phenotype of HPMCs were found to be positively related to epithelial-mesenchymal transition progress and PM fibrosis ex vivo in 93 patients who underwent continuous ambulatory peritoneal dialysis (PD), and also in $\mathrm{HG}$-induced immortal HPMCs and an animal model of PD. Evidence from chromatin immunoprecipitation and luciferase reporter assays supported that $Y B X 1$ is transcriptionally regulated by the direct binding of Twist to E-box. Overexpression of Twist and YB-1 led to an increase in epithelial-mesenchymal transition, proliferation, and cell cycle progress of HPMCs, which might contribute to PM fibrosis. In contrast, the silencing of Twist or YB-1 inhibited HG-induced growth and cell cycle progression of HPMCs; this led to a down-regulation in the expression of cyclin Ds and cyclin-dependent kinases, finally inhibiting PM fibrosis. Twist contributes to PM fibrosis during PD treatment, mainly through regulation of YB-1. (Am J Pathol 2015, 185: 2181-2193; http://dx.doi.org/10.1016/j.ajpath.2015.04.008)
\end{abstract}

Peritoneal dialysis (PD) is a common life-sustaining treatment in patients with end-stage renal disease, which uses the singlecell peritoneal membrane (PM) as a semipermeable membrane. ${ }^{1}$ Sometimes, this dialysis is broken off by PM injury and fibrosis, which are involved in the morphological alterations of the peritoneum in patients who undergo chronic dialysis. It has been increasingly recognized that the phenotypic transition of the human peritoneal mesothelial cells (HPMCs) is a key step in PM early injury and fibrosis. Previous work also showed that a high glucose $(\mathrm{HG})$ injury was actually responsible for fibrosis of the PM. ${ }^{2}$

An early process in peritoneal fibrosis is epithelialmesenchymal transition (EMT) of HPMCs, ${ }^{3}$ which is a complex, step-wise phenomenon detrimental in fibrogenic tissues. $^{4,5}$ It was thought that soon after dialysis is initiated, peritoneal mesothelial cells undergo a transition from an epithelial phenotype to a mesenchymal phenotype, with EMT markers changed after 48 hours of $\mathrm{HG}$ stimulation in vitro. HG-induced phenotypic transition was the earliest phenomenon of peritoneal damage and could be observed earlier than significant morphological changes of HPMC cells. Although peritoneal fibrosis was an intractable process, a significant reversal of EMT of HPMCs at early damage stage would be found.

Therefore, preventing EMT can ameliorate peritoneal fibrosis, preserving the PM during PD. Some researchers

Supported in part by the National Scientific Foundation of China grants 81270849 (L.H.), 81270768 (H.W.), and 2011BAI10B08 (S.S.).

L.H., M.C., J.H., and S.L. contributed equally to this work.

Disclosures: None declared. 
have found that the growth and proliferation of HPMCs significantly changes when fibrosis develops in the PM after HG stimulation, followed by the cell cycle progression. ${ }^{6}$ Cell proliferation could not be affected or even be inhibited at the initial stimulation time for 48 hours, and then had an abnormal proliferation and cell cycle progress at the following HG stimulation days. Thus, the inhibition of growth and the induction of cell cycle arrest of HPMCs might contribute to protection against PM fibrosis.

Twist, a highly conserved helix-loop-helix transcription factor, was newly identified as a key regulator of embryogenesis, pulmonary fibrosis, and cancer metastasis. ${ }^{7-9}$ Our previous study found that Twist was involved in hypoxia-induced EMT of human tubular epithelial cells and might contribute to the pathogenesis of progressive renal fibrosis. ${ }^{10}$ Previous work in our laboratory also initially revealed the role of Twist in peritoneal fibrosis in vitro. ${ }^{11}$ It implied that HG stimulation could activate Twist, and promote EMT of HPMCs in vitro. ${ }^{12}$ However, the precise role of Twist in peritoneal fibrosis in vivo and its underlying mechanism are not fully understood.

YB-1, a member of the Y-box-binding protein family, contains a highly conserved nucleic acid binding domain and has several E-boxes located in the core promoter. ${ }^{13}$ YB-1 has been proposed to possess various biological activities in both nucleus and cytoplasm. ${ }^{14}$ Thus, YB-1, which is up-regulated in both proliferating adult tissues and cancer cells, may be closely correlated with the proliferation and phenotypic transition of HPMCs. Previous work in tumor showed that YB-1 might be a main target of Twist, and found that YB-1 reexpression almost completely rescued Twist siRNA-induced growth arrest, but cotransfection of Twist expression plasmid could not rescue YB-1 siRNA-induced growth arrest. ${ }^{15}$ Although YB-1 is thought to be a major downstream target of Twist and necessary for tumor cell proliferation and cell cycle progress, the mechanism of Twist and YB-1 in proliferation of HPMCs and EMT-induced peritoneal fibrosis were still unclear. In this study, we continue to uncover the role of Twist and YB-1 in proliferation and cell cycle progress of HPMCs and further investigate the exact mechanism of PM fibrosis.

\section{Materials and Methods}

\section{Ethics Statement}

The study protocol conformed to the ethical guidelines of the 1975 Declaration of Helsinki and was approved by the ethics committee of Xijing Hospital (Xi'an, Shaanxi, People's Republic of China). Written informed consent was obtained from each patient. The Ethics Committee for Animal Experiments of the Fourth Military Medical University (Xi'an, Shaanxi, People's Republic of China) approved all animal work (permit number 20120023), and the experimental protocols strictly complied with the institutional guidelines and the criteria outlined in the Guide for the Care and Use of Laboratory Animals. $^{16,17}$

\section{HPMC Isolation and Culture}

HPMCs, which were obtained from the effluents of 93 patients undergoing continuous ambulatory PD (CAPD) at our PD center in the Department of Nephrology, Xijing Hospital, from January 1, 2013, to December 31, 2013, were collected and cultured according to a previously described method. ${ }^{16,18-20}$ The use of human peritoneal fluid in this study was approved by the Institutional Review Board of the Fourth Military Medical University. This study was conducted in accordance with international guidelines for the use of human tissues. The inclusion criteria were as follows: $<65$ years of age, duration of CAPD $>1$ month, no peritonitis within the past 6 months, the use of $1.5 \%$ glucose dialysis solution, and no history of abdominal surgery or long-term use of an immunosuppressant. The CAPD patients were sorted into four groups $(\mathrm{G})$ according to the duration of dialysis $\left(G_{1}\right.$ was 1 to 6 months; $G_{2}, 7$ to 24 months; $\mathrm{G}_{3}, 25$ to 48 months; and $\mathrm{G}_{4},>48$ months) (Supplemental Table S1). The human immortal peritoneal mesothelial cell line HPMC (American Type Culture Collection, Rockville, MD) is an untransformed HPMC line that yields data similar to the results obtained with primary cells. The immortal and primary HPMC cell lines were cultured at $37^{\circ} \mathrm{C}$ with $5 \% \mathrm{CO}_{2}$ in Earle's M199 medium ${ }^{21}$ supplemented with $10 \%$ fetal calf serum (Gibco, Grand Island, New York), $100 \mathrm{U} / \mathrm{mL}$ penicillin, and $0.1 \mathrm{mg} / \mathrm{mL}$ streptomycin. EMT was induced in immortal HPMCs with HG $(60 \mathrm{mmol} / \mathrm{L}$; R\&D Systems, Minneapolis, MN), as described previously. ${ }^{14,20,22}$

\section{Plasmid Construction and Cell Transfection}

The pcDNA3.1-Twist plasmid was a gift from Prof. Carlotta A. Glackin (National Medical Center and Beckman Research Institute, Durate, CA). ${ }^{10}$ Targeted siRNA was determined with the siRNA design tool (Invitrogen, Carlsbad, CA). The Twist siRNA-specific targeting sequence was 5'-AAGATGGCAAGCTGCAGCTAT-3'; a scramble sequence, 5'-TCAGCAAGGTGACTGAGAC-3', was used as control. The YB-1 siRNA-specific target sequence was 5'-GGCGAAGGUUCCCACCUUATT-3'; 5'-UUCUCCGAACGUGUCACGUdTdT- $3^{\prime}$ was used as a control. The transfections were performed with Lipofectamine 2000 (Invitrogen), according to the manufacturer's instructions. Real-time PCR or Western blot analysis was used to assess the transfection efficiency in cells harvested 48 hours for mRNA and 72 hours for protein after siRNA transfection.

Generation of Twist Overexpression, Twist-shRNA, and YB-1-shRNA Lentivirus

The Twist overexpression, Twist-shRNA, or YB-1 shRNAspecific targeting sequence and the control sequence were described as above. Human embryonic kidney $293 \mathrm{~T}$ cells (Invitrogen) were transfected with the two expression vectors PLVX-IRES-mCherry and PLVX-shRNA2 and two packaging plasmids PMD and psPAX2, at 4,1 , and $3 \mathrm{mg}$ of 
DNA per 150-mm plate. After 6 hours, Dulbecco's modified Eagle's medium was changed. Forty-eight hours later, supernatants from four plates were pooled, filtered, and stored at $4^{\circ} \mathrm{C}$ short-term. Lentivirus titers were determined by transfecting human embryonic kidney $293 \mathrm{~T}$ cells with a dilution series of the viral suspension, and lentivirus samples with a titer of $4 \times 10^{8}$ transfection units $/ \mathrm{mL}$ were stored at $-80^{\circ} \mathrm{C}^{23,24}$

\section{Peritoneal Exposure Model in Rats}

A chronic infusion model of nonuremic animal PD was used, as described previously. ${ }^{6,14}$ Briefly, 16 male Sprague-Dawley rats (weight, 250 to $300 \mathrm{~g}$ ) were divided into two groups with eight rats in each group. The control group was injected with normal saline $(100 \mathrm{~mL} / \mathrm{kg}$ per day) each day for 6 weeks; the HG-PD fluid (PDF; pH 4.5 to 6.5; GDP, 4.25\%; Baxter, Chicago, IL) group was injected with $4.25 \%$ glucose dialysis solution (100 $\mathrm{mL} / \mathrm{kg}$ per day) each day for 6 weeks. Another $24 \mathrm{HG}-\mathrm{PDF}$ male Sprague-Dawley rats were divided into three groups, which were separately treated with empty lentivirus vector (control-shRNA), lentivirus vector containing Twist-shRNA (Twist-shRNA), or YB-1 shRNA (YB-1-shRNA) i.p. via the peritoneal access port at a dose of $4 \times 10^{8}$ transfection units every 5 days, three times during HDF-PD injection. ${ }^{25}$ Rats were manipulated and cared for according to NIH Animal Care and Use Committee guidelines in the Experiment Animal Center of the Fourth Military Medical University (Xi'an, Shanxi, Province, People's Republic of China).

\section{Cell Proliferation Assay (MTT Assay)}

HPMCs were plated onto 96-well plates (Corning, NY) at a density of $2.5 \times 10^{4}$ cells per well. Viable cells were assayed at 1, 2, 3, 4, and 5 days. After incubation with MTT assay (Sigma, San Francisco, CA), the cells were lysed in $150 \mu \mathrm{L}$ of $100 \%$ dimethyl sulfoxide and the absorbance was tested at $490 \mathrm{~nm}$ with a 96-well plate reader (Dynex Technologies, Chantilly, VA). ${ }^{24}$ Each experiment was performed in triplicate.

\section{Flow Cytometry}

Flow cytometric analysis was performed as previously described. ${ }^{24}$ The transfected cells were harvested when they were $70 \%$ to $80 \%$ confluent. After washing in ice-cold phosphate-buffered saline, the cells were suspended in approximately $0.5 \mathrm{~mL}$ of $70 \%$ alcohol and kept at $4^{\circ} \mathrm{C}$ overnight. The DNA content of stained nuclei was analyzed by a flow cytometer (EPICS XL; Coulter, Miami, FL). The cell cycle was analyzed with FACScan software version 1.4 (Becton Dickinson, San Jose, CA). The proliferous indices (PIs) were calculated with the following equation:

$$
\mathrm{PI}=\left(\mathrm{S}+\mathrm{G}_{2}\right) /\left(\mathrm{S}+\mathrm{G}_{2}+\mathrm{G}_{1}\right)
$$

Each experiment was performed in triplicate.

\section{RT-PCR}

Total RNA was extracted with the RNeasy kit (Qiagen GmbH, Hilden, Germany), and cDNA was obtained from $500 \mathrm{ng}$ of total RNA with an Omniscript RT kit (Qiagen $\mathrm{GmbH}$ ). Quantitative PCR was performed in a LightCycler (Roche Diagnostics GmbH, Mannheim, Germany) with a SYBR Green kit (Roche Diagnostics $\mathrm{GmbH}$ ). The following specific primer sets were used: glyceraldehyde-3-phosphate dehydrogenase, $5^{\prime}$-CCTCAAGATCATCAGCAAT-3' (forward) and 5'-CCATCCACAGTCTTCTGGGT-3' (reverse; used for normalization); Twist, 5'-CGGGAGTCCGCAGTCTTA-3' (forward) and 5'- ACCGGGTTGGTTTTGATCTG-3' (reverse); CDH1 (E-cadherin), 5'-TTCCCTCGACACCCGATTC-3' (forward) and 5'-TAGGTGGAGTCCCAGGCGTA- $3^{\prime}$ (reverse); $\alpha$-smooth muscle actin ( $\alpha$-SMA), $5^{\prime}$-AAGATGACCCAGATCATGTT-3' (forward) and $5^{\prime}$-TCATAGATGGGGACATTGT-3' (reverse); and YB-1, 5'-AAGTGATGGAGGGTGCTGAC- $3^{\prime}$ (forward) and $5^{\prime}$-TTCTTCATTGCCGTCCTCTC- $3^{\prime}$ (reverse). Experiments were performed in duplicate. After amplification, the PCR products were confirmed by melt curve analysis and gel electrophoresis.

\section{Western Blot Analysis}

Total protein extracts from the cultured cells were loaded onto SDS-PAGE gels, and Western blot analysis was performed according to a standard protocol. The antibodies used were as follows: anti-Twist, diluted 1:100 (Sigma); anti-E-cadherin, anti- $\alpha$-SMA, and anti-YB-1, diluted 1:50 (Santa Cruz Biotechnology, Dallas, TX); anti-cyclin D1, anti-cyclin E, anti-cyclin-dependent kinase (CDK) 2, anti-CDK4, antiproliferating cell nuclear antigen (PCNA), anti-matrix metalloproteinase (MMP)-2, anti-MMP-9, and anti-p27, diluted 1:150 (Santa Cruz Biotechnology); and anti- $\beta$-actin (Sigma), diluted 1:4000. The staining was performed as described previously. $^{24}$

\section{Immunofluorescence and Immunohistochemistry}

Cultured cells were grown on $0.8 \times 0.8$-cm slides, rinsed with ice-cold phosphate-buffered saline, and fixed in $4 \%$ paraformaldehyde. Immunofluorescence or immunohistochemistry staining was performed on cultured cells or paraffin-embedded sections, as described previously. ${ }^{12}$ The samples were incubated with $\mathrm{Ms} / \mathrm{Rb}$ polyclonal anti-Twist (Abcam, Cambridge, England; dilution 1:100), anti- $\alpha$-SMA, anti-PCNA, and anti-E-cadherin (Santa Cruz Biotechnology; dilution 1:200), anti-fibronectin (FN), or anti-YB-1 antibodies (Santa Cruz Biotechnology; dilution 1:100). Then, they were counterstained with fluorescein isothiocyanate-labeled IgG secondary antibodies (Invitrogen) or poly-horseradish peroxidase anti-Ms or $\mathrm{Rb}$ IgG secondary antibody (ZSBiO, Beijing, People's Republic of China) and were stained with diaminobenzidine. Images were obtained with a Leica TCS microscope and imaging software (Leica software version SP8; Leica 
A

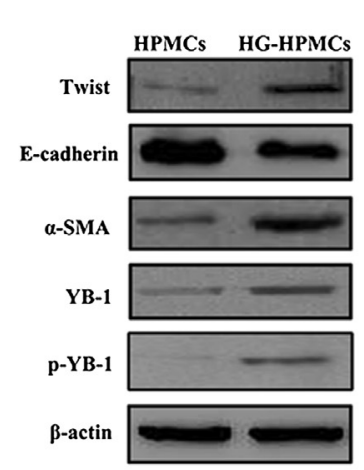

B

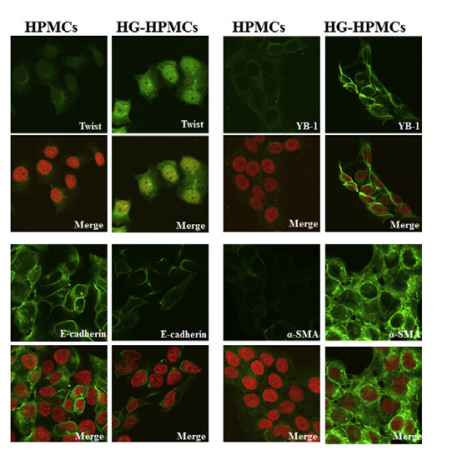

C

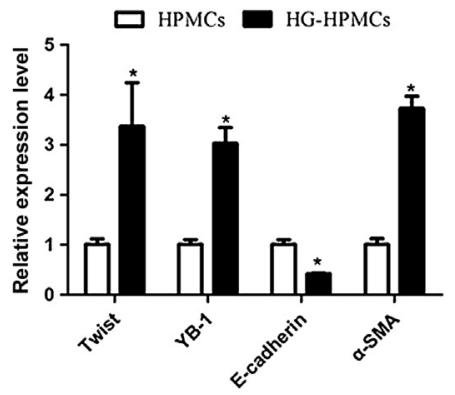

D
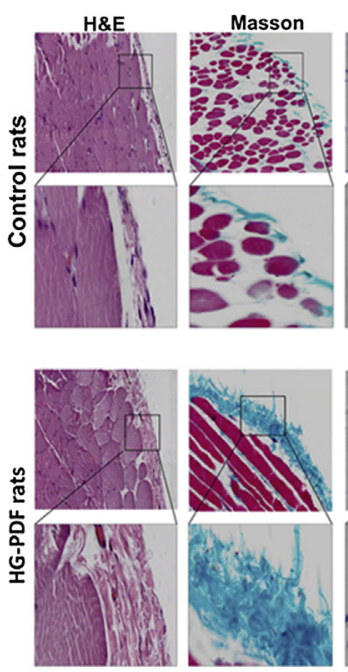

E

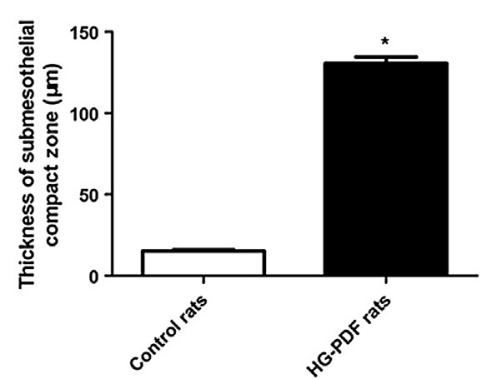

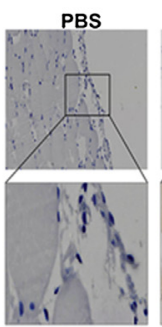
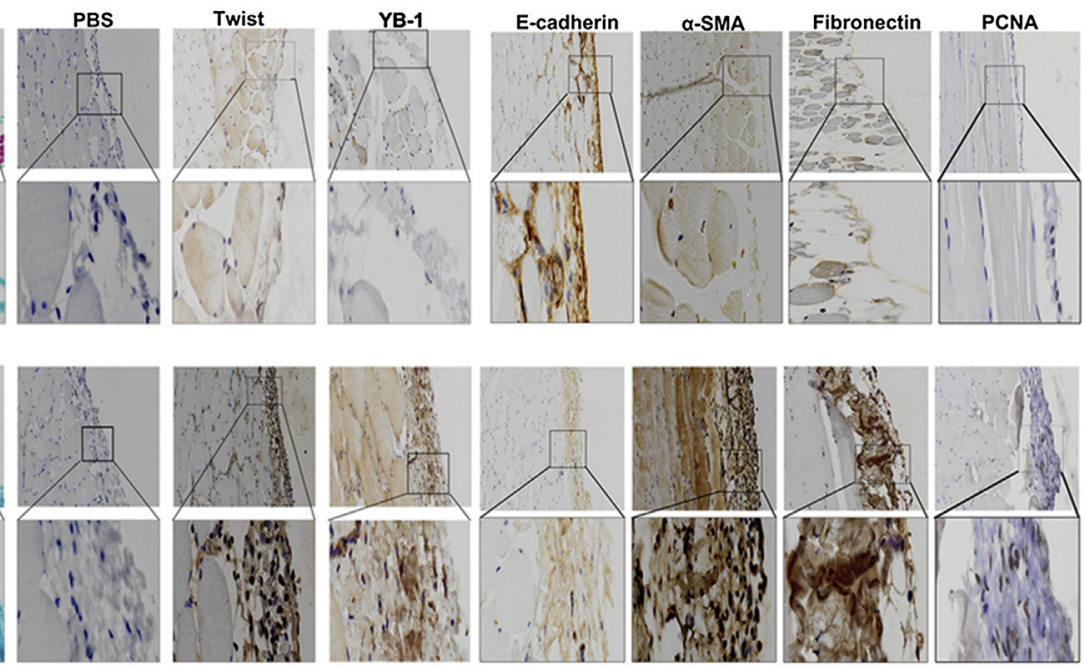

$\mathbf{F}$

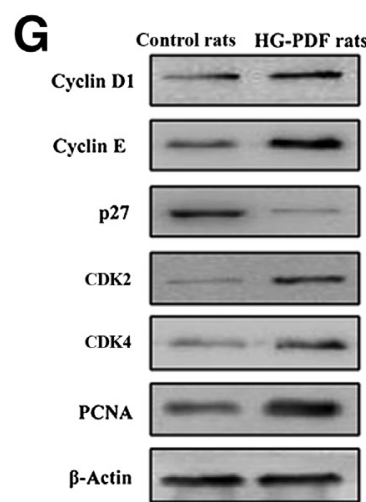

Figure 1 Twist and YB-1 expression in high glucose (HG)-induced immortal human peritoneal mesothelial cells (HPMCs) in vitro. A: Western blot analysis shows the induction of Twist, YB-1, pYB-1, E-cadherin, and $\alpha$-smooth muscle actin ( $\alpha$-SMA) expression in immortal HPMCs, which were exposed to HG for 48 hours in vitro. B: Fluorescence microscopy illustrates the location and expression of Twist, YB-1, E-cadherin, and $\alpha$-SMA in HG-induced immortal HPMCs exposed to HG for 48 hours. C: Real-time PCR analysis shows the level of Twist, YB-1, E-cadherin, and $\alpha$-SMA induced by HG stimulation for 48 hours in immortal HPMCs. Bars represent the fold induction over untreated cells. D: Staining for the Twist, YB-1, fibronectin (FN), proliferating cell nuclear antigen (PCNA), and Masson in peritoneal samples by immunohistochemistry reveals that peritoneal dialysis (PD) fluid containing HG exposure (HG-PDF) induces the up-regulation of Twist, YB-1, and PD-induced peritoneal membrane (PM) fibrosis in vivo. E: The histogram shows the thickness for hematoxylin and eosin (H\&E) staining in the submesothelial compact zone in HG-PDF rats $(130.63 \pm 11.26 \mu \mathrm{m})$ compared with control rats $(15.25 \pm 2.43 \mu \mathrm{m})$. F: The histogram shows the average of cell cycle analysis in omentum-derived HPMCs from HG-PDF rat $(64.82 \% \pm 2.41 \%)$ compared with the omentum-derived HPMCs from control rats $(47.15 \% \pm 5.00 \%)$. Each was conducted in triplicate. G: Western blot analysis shows the expression of cyclin D1, cyclin E, p27, cyclin-dependent kinase (CDK) 2, CDK4, and PCNA in omentum-derived mesothelial cells from HG-PDF compared with the cells from control rats. Data are given as means \pm SEM (C and $\mathbf{E}) . n=3$ independent experiments conducted in duplicate $(\mathbf{C}) ; n=8(\mathbf{E})$. ${ }^{*} P<0.05$ versus controls. Original magnifications: $\times 200$ (B); $\times 100$ (D, top panels); $\times 400$ (D, bottom panels).

Microsystems, Wetzlar, Germany). The images were processed with Photoshop CS (Adobe, San Jose, CA). The positive expression was graded from 0 to $3+(0$, no staining; $1+, 1 \%$ to $25 \% ; 2+, 26 \%$ to $50 \% ; 3+,>50 \%$ staining).

\section{ChIP}

Chromatin immunoprecipitation (ChIP) was performed as previously described. ${ }^{10,25}$ Chromatin fragments were 
Table 1 Expression and Location of Twist in Primary HPMCs

\begin{tabular}{|c|c|c|c|c|c|c|}
\hline \multirow[b]{2}{*}{ Morphological features of primary HPMCs } & \multicolumn{4}{|c|}{ CAPD times (months) } & \multirow[b]{2}{*}{ Fisher's $\chi^{2}$} & \multirow[b]{2}{*}{$P$ value } \\
\hline & $\begin{array}{l}1-6 \\
(n=37)\end{array}$ & $\begin{array}{l}7-24 \\
(n=35)\end{array}$ & $\begin{array}{l}25-48 \\
(n=18)\end{array}$ & $\begin{array}{l}>48 \\
(n=3)\end{array}$ & & \\
\hline Mixed & 9 & 22 & 3 & 0 & & \\
\hline Fibroblast-like & 0 & 7 & 14 & 3 & 64.950 & $<0.001$ \\
\hline
\end{tabular}

CAPD, continuous ambulatory peritoneal dialysis; HPMC, human peritoneal mesothelial cell.

co-immunoprecipitated from HG-treated HPMCs and control lysates with the appropriate amount of Twist antibody or an equivalent amount of rabbit $\operatorname{IgG}(8 \mu \mathrm{g})$ as a control. The purified DNA fragments were used as templates for PCR amplification. Real-time PCR was performed with an ABI 7900 Sequence Detection System (Applied Biosystems, Carlsbad, California) and a SYBR Green PCR core reagent kit (Applied Biosystems). The specific primers were as follows: YB-1 ChIPF1, 5'-AGAAACTGACTCGCGAACTATTC-3'; YB-1 ChIPR1, 5'-CAACTCACATTTATTGAACAAG- $3^{\prime}$. The values were normalized to the input and expressed as a fold increase relative to the $\mathrm{IgG}$ control.

\section{Transient Transfection Luciferase Assay}

The YB-1 promoter region from -1 to -1000 was cloned into the pGL3 luciferase vector (Promega, Madison, WI). The primers used to generate the mutant reporter were as follows: mutYB-1, 5'-GTGGATAGTAATCCCTCTATTGTAGAGCTGTTGCAGGAATAAAGTG-3' (forward) and 5'-CACTTTATTCCTGCAACAGCTCTACAATAGAGGGATTACTATCCAC- $3^{\prime}$ (reverse). For the transient transfection assays, HG-treated HPMCs or control cells were seeded 48 hours before transfection. Then, pGL3-basic was transfected with the PRLsv40 plasmid into cells in the presence of Lipofectamine 2000. The luciferase activity in the cell lysates that were collected 72 hours after transfection was measured as relative light units with the Fluoroscan Ascent FL (Labsystems, Franklin, MA) and the Dual-Luciferase Assay System (Promega). The relative luciferase activity was defined as the ratio of the firefly luciferase activity/mean Renilla luciferase activity for each construct relative to the pGL3 control vector (Promega).

\section{Statistical Analysis}

The bands from the Western blot or real-time PCR were quantified by Quantity One software version 4.62 (BioRad, Hercules, CA). The numerical data are presented as the means \pm SEM, and SPSS software version 12.0 (SPSS Inc., Chicago, IL) was used for statistical analysis. The differences between the means were assessed using $t$-test, an analysis of variance, or the $\chi^{2}$ test. Significant differences were determined by an analysis of variance, followed by post hoc comparisons with the Fisher protected least-significant-difference test. $P<$ 0.05 was considered statistically significant.

\section{Results}

The Up-Regulation of Twist and YB-1 0ccurs in Immortal HPMCs and in Animal Models

In our previous in vitro work, we found that Twist was overexpressed in immortal HPMC cell lines. ${ }^{11}$ To obtain direct evidence of the effects of Twist and YB-1 expression on HGinduced EMT, we tested the expression of Twist and YB-1 genes in HG cultured immortal HPMCs in vitro by Western blot analysis and real-time PCR. When HPMCs were incubated with $\mathrm{HG}$ medium ( $60 \mathrm{mmol} / \mathrm{L}$ glucose) for 48 hours, the cells lost intercellular junctions, became scattered, and prepared to transform into spindled fibroblastic-shaped cells. Concordant with our ex vivo results, HPMCs demonstrated a loss of Ecadherin expression, a gain in $\alpha$-SMA expression, translocation of Twist from the cytoplasm to the nucleus, and an increase in Twist and YB-1 expression at both the protein and mRNA levels in response to $\mathrm{HG}$ stimulation (Figure 1, A-C). Twist translocated into nucleus, but YB-1 only partly translocated into the nucleus after $\mathrm{HG}$ stimulation.

We also established an animal model of PD in which rats were injected with HG-PDF (4.25\% glucose dialysis solution) or normal saline solution over a period of 6 weeks (Figure 1, D-F). The loss of the mesothelial cell monolayer with a thicker PM (130.63 \pm 11.26$)$ was found in the parietal peritoneum from the untreated PD group compared with the saline-treated control group (15.25 \pm 2.43$)$. An analysis of Twist, YB-1, E-cadherin, $\alpha$-SMA, FN, and PCNA by immunohistochemistry indicated that HG-PDF up-regulated Twist, YB-1, $\alpha$ SMA, FN, and PCNA and down-regulated E-cadherin in the PM of our animal model of PD. Masson staining and overexpression of FN, PCNA, and EMT markers $\alpha$-SMA showed that HG-PD fluid induced PM fibrosis, rapid proliferation, and EMT of HPMCs in the PD animal model. Cell cycle analysis was used to test the cell cycle progress of omentum-derived mesothelial cells from the PD animal model by laser-scanning flow cytometry (Figure 1, E-G). HPMCs from HG-PDF rats showed a significant decrease in the proportion of HPMCs in the $\mathrm{G}_{1}$ phase from $64.82 \% \pm 2.41 \%$ to $47.15 \% \pm 5.00 \%$. PI increased from $35.18 \%$ to $52.85 \%$. A substantial increase in the proportion of cells in the $\mathrm{S}$ fraction was also observed in HPMCs from HG-PDF rats. These data showed that both HGinduced HPMCs and omentum-derived HPMCs from HG-PDF rats overexpressed Twist and YB-1 on increased cell proliferation, progressed to the $G_{1} / S$ cell cycle, and induced fibrosis. 


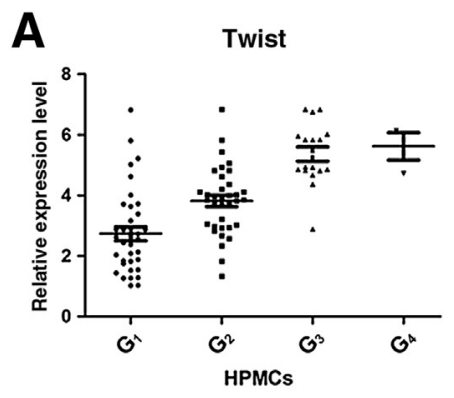

B $\quad$ YB-1

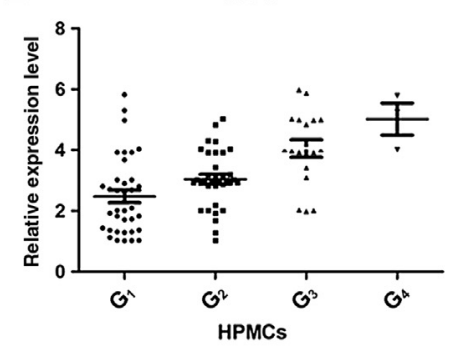

c

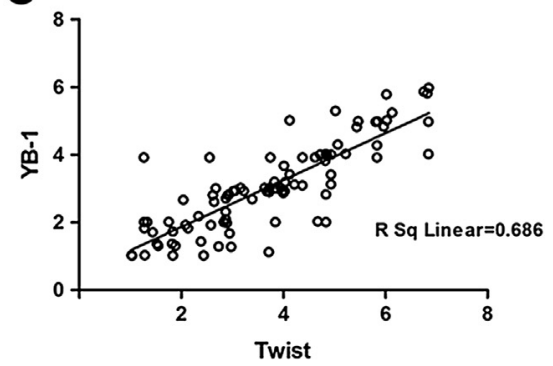

Figure 2 Ex vivo expression of Twist and YB-1 in peritoneal dialysis (PD) effluent-derived human peritoneal mesothelial cells (HPMCs). A and B: Real-time PCR showing the mRNA levels of Twist and YB-1 in effluentderived HPMCs from 93 patients from the different groups $\left(G_{1}\right.$ to $\left.G_{4}\right)$ that underwent PD treatment. C: The correlation analysis of Twist and YB-1 in effluent-derived HPMCs from patients from different groups $\left(G_{1}\right.$ to $\left.G_{4}\right)$ that underwent PD treatment.

The Up-Regulation of Twist and YB-1 Occurs ex Vivo in HPMCs from Patients with CAPD

To better understand the role of Twist and YB-1 in HPMCs induced by $\mathrm{HG}$ injury in vivo, we investigated the expression levels of Twist and YB-1 by real-time PCR in effluent-derived HPMCs ex vivo from effluents of 93 patients with CAPD who were followed up during a return visit from January 1 to December 31, 2013. After PD treatment, the morphological features of HPMCs were markedly variable and ranged from a cobblestone-like appearance to mixed cell populations and fibroblast-like cells (Table 1), which is consistent with previous findings. ${ }^{5,17,20}$ We assessed Twist and YB-1 expression in all 93 PD patients who were grouped into $G_{1}, G_{2}, G_{3}$, and $G_{4}$ groups according to the interval of $\mathrm{PD}$ treatment time. We found that Twist expression significantly increased in HPMCs from patients with CAPD in $\mathrm{G}_{2}, \mathrm{G}_{3}$, and $\mathrm{G}_{4}$, compared with patients in $\mathrm{G}_{1}$; this was consistent with previous in vitro studies (Figure 2A). ${ }^{11} \mathrm{We}$ also found the highest YB-1 expression in patients in $\mathrm{G}_{2}, \mathrm{G}_{3}$, and $\mathrm{G}_{4}$ who underwent more than half a year of PD compared with patients with CAPD who underwent PD for a shorter period of time $\left(\mathrm{G}_{1}\right)$ (Figure $\left.2 \mathrm{~B}\right)$. The $\mathrm{YB}-1$ gene, which positively correlated with Twist, may be related to HGinduced injury of the PM during long-term PD, just like Twist $(r=0.686, P<0.01)$ (Figure 2C). Thus, Twist and YB-1 may be related to the transition of HPMCs after the injury of PMs by exposure to HG during long-term PD.

\section{Twist Transcriptionally Regulates YB-1 Expression by Directly Binding the E-Box in YB-1 Promoter}

Structural analysis has revealed the existence of several E-boxes within the YB-1 promoter, suggesting a direct regulation of YB-1 transcription by Twist. To address this hypothesis, ChIP was performed to examine the binding of Twist to the E-box element in the YB-1 promoter in HPMCs. One E-box site (CACGTG, -654 to -649 ) in the promoter region of the YB-1 gene was predicted to be a candidate binding site of Twist. A 50-fold enrichment was set as the threshold for positive Twist binding. Our results demonstrated strong binding of Twist at this E-box site in HG-induced HPMCs, but weak binding in normal HPMCs. These data suggested that the greatly increased binding signal in the HG-induced HPMCs might be in agreement with the higher Twist expression (Figure 3A).

To further confirm the binding of Twist to the E-box element in the YB-1 promoter, a luciferase reporter assay was performed. Transfected full-length YB-1 plasmids containing an E-box site into HPMCs could stimulate luciferase activity either in normal medium $(P=0.006)$ (Figure $3 \mathrm{~B})$ or high-glucose medium $(P=0.007)$ (Figure $3 \mathrm{C})$ compared with control vectors. The effect was more significant in HG-stimulated HPMCs (Figure 3C). Mutation or deletion of the E-box moderately affected their response, which was statistically significant compared with the YB-1 plasmids, especially in HG-HPMCs (mutation, $P=0.006$; deletion, $P=0.005$ ). These results suggested that the activation of Twist-YB-1 signaling occurs via the binding of Twist to YB-1, predominantly at the E-box site in its promoter region.

\section{The Down-Regulation of Twist Causes Growth Retardation and $\mathrm{G}_{1} / \mathrm{S}$ Arrest in HG-Induced HPMCs}

To obtain further insight into the biological relevance of both Twist and YB-1, we analyzed the effect of Twist on the fibrosis, proliferation, and cell cycle progression of immortal HPMCs after HG stimulation. Cells were HG stimulated and cultured after transfection, which mimicked the damage and treatment of PDF in PD patients. Western blot analysis and immunofluorescence showed that Twist, YB-1, and $\alpha$-SMA protein levels significantly decreased, whereas E-cadherin protein levels significantly increased, after transfection of siRNA against Twist in HG-induced HPMCs or in transfected HPMCs, which were stimulated by HG (Figure 4, A and B). But Twist and YB-1 were not rescued by HG after knockout of Twist in HPMCs (Supplemental Figure S1). Real-time PCR 

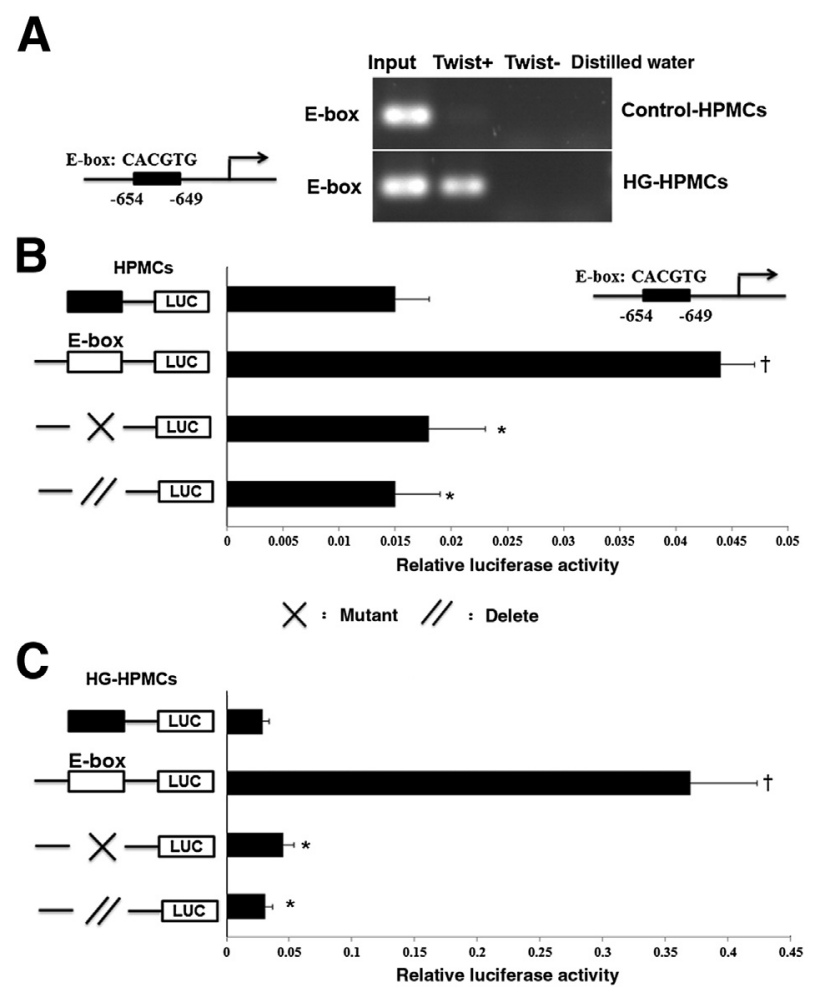

Figure 3 Twist directly induces the expression and transcriptional activation of YB-1 in human peritoneal mesothelial cells (HPMCs). A: The identification of an E-box (CACGTG) in the YB-1 promoter from -654 to -649 . Chromatin immunoprecipitation was used to examine the binding of Twist to the YB-1 promoter in high glucose (HG)-induced HPMCs and in control cells. Reaction controls included immunoprecipitation performed with a nonspecific IgG monoclonal antibody; PCR was performed with whole-cell genomic DNA (input). A representative example of three independent experiments is shown. B and C: For the competition assays with the E-box, 200-fold molar excess of the YB-1 plasmid, mutant E-box, and deleted E-box were used in HPMCs (B) and HG-induced HPMCS (C). The average relative luciferase activity data in normal HPMCs transfected with control plasmid, YB-1 with normal E-box, mutant E-box, and E-box deleted separately are $0.015 \pm 0.003$, $0.044 \pm 0.003,0.018 \pm 0.005$, and $0.015 \pm 0.004$, respectively. All results shown are representative of at least three independent experiments. The average data in HG-induced HPMCs transfected with control plasmid, YB-1 with normal E-box, mutant E-box, and E-box deleted separately are $0.029 \pm 0.005$, $0.037 \pm 0.053,0.045 \pm 0.009$, and $0.031 \pm 0.006$, respectively. ${ }^{*} P<0.05$ versus YB-1 (E-box) plasmid; ${ }^{\dagger} P<0.05$ versus control plasmid.

results were consistent with the immunofluorescence and Western blot analysis (Figure 4C). These experiments demonstrated that the down-regulation of Twist could inhibit EMT in HG-induced HPMCs. MTT assays showed the apparent growth retardation of HG-induced HPMCs after Twist expression was knocked down in HG-induced HPMCs (Figure 4D), which means that down-regulated Twist could hinder the proliferation of HG-induced HPMCs. To understand the implications on the cell cycle, HG-HPMCs were treated with Twist-siRNA at 72 hours after transfection and assayed for cell cycle analysis by laser-scanning flow cytometry (Figure 4, E-G). The silencing of Twist expression led to a significant increase in the proportion of HPMCs in the $\mathrm{G}_{1}$ phase, from $57.22 \% \pm 1.99 \%$ to $82.03 \% \pm 1.63 \%$. PI decreased from $42.78 \%$ to $17.97 \%$. Furthermore, we observed a substantial decrease in the proportion of cells in the S fraction. All of these results showed that the knockdown of Twist also down-regulated the expression of YB-1, which resulted in the inhibition of cellular proliferation, $\mathrm{G}_{1} / \mathrm{S}$ cell cycle arrest, and the delay of EMT-induced fibrosis. Thus, Twist might be an essential mediator of regulation of growth and proliferation in HG-induced HPMCs.

We also established PD animal model treatment with Twist shRNA lentivirus compared with control shRNA lentivirus (Figure 4, H and I). A thinner PM was found, with $61.50 \pm 23.57 \mu \mathrm{m}$ in the parietal peritoneum in TwistshRNA PD rats compared with $121.50 \pm 14.34 \mu \mathrm{m}$ in the control-shRNA group (Figure 4H). An analysis of Twist, YB-1, E-cadherin, $\alpha$-SMA, FN, and PCNA by immunohistochemistry indicated that treatment of Twist-shRNA lentivirus in PD rats caused down-regulation of YB-1, $\alpha$-SMA, FN, and PCNA, but up-regulation of E-cadherin in the PM. Decreased expression of FN, PCNA, and EMT marker $\alpha$-SMA showed that the down-regulation of Twist contributed to the alleviation of PM fibrosis, slower peritoneal transport rate [dialysate-to-plasma rate of urea (D/P urea) and end-to-initial dialysate (D/D0) glucose] for lower molecular weight (Supplemental Figure S2), and growth retardation of HPMCs in the studied PD animal model.

The Up-Regulation of Twist Increases the Expression of YB-1, Promotes HG-Induced Fibrosis, and Leads to Abnormally Rapid Growth

We further induced the up-regulation of Twist expression to study the role of Twist in the proliferation and fibrosis of HPMCs. Western blot analysis and immunofluorescence showed that the overexpression of Twist after transfection of the full-length human Twist plasmid into immortal HPMCs led to significant down-regulation of E-cadherin and up-regulation of Twist, YB-1, and $\alpha$-SMA protein (Figure 5, A and B) as well as mRNA (Figure 5C) levels. This is consistent with the results from HG-induced primary cultured HPMCs. These data suggested that the overexpression of Twist could up-regulate YB-1 expression and prompt EMT-induced fibrosis. The results of the MTT assays showed that when Twist was up-regulated by transfection of a plasmid, significant and rapid growth of HPMCs was observed from the third day compared with the cells that were transfected with a control plasmid (Figure 5D). To understand the implications of this increase in growth rate, the cells were treated with pcDNA3.1-Twist and assayed for DNA content at 72 hours after transfection by laser-scanning flow cytometry (Figure 5, E-G). The up-regulation of Twist expression also led to a significant and reproducible decrease in the proportion of HPMCs in the $\mathrm{G}_{1} / \mathrm{S}$ phase; in addition, the percentage of cells in $\mathrm{G}_{1}$ phase decreased from $56.64 \% \pm 2.16 \%$ to $42.18 \% \pm 2.19 \%$. Furthermore, we observed a substantial increase in the proportion of cells in the $S$ phase. PI staining also increased from $43.36 \%$ to $57.82 \%$. Therefore, Twist regulated YB-1 expression by promoting 

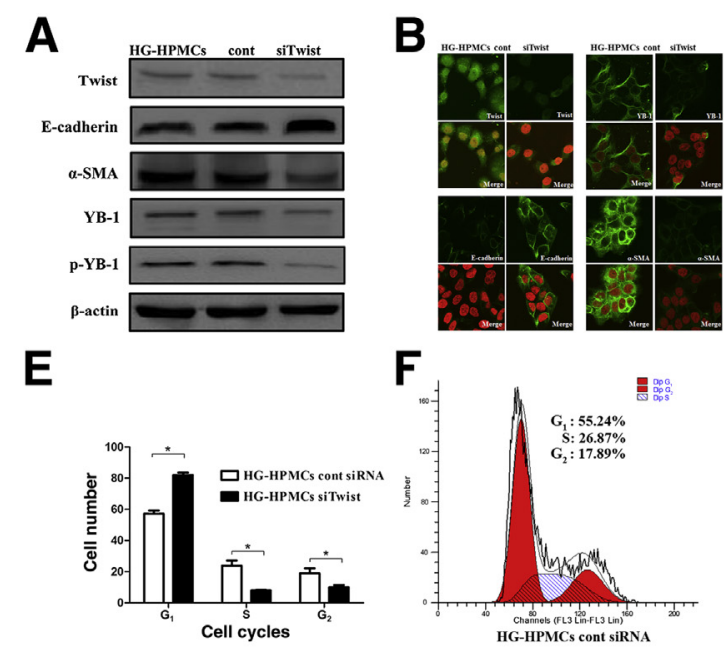

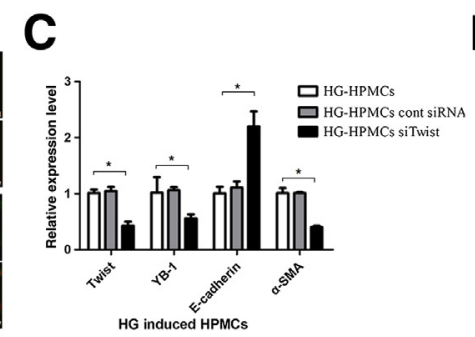

G

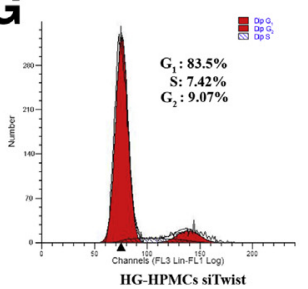

D

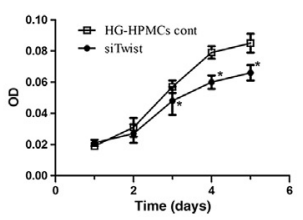

H

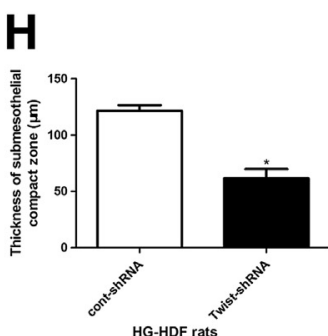

HG-HDF rats

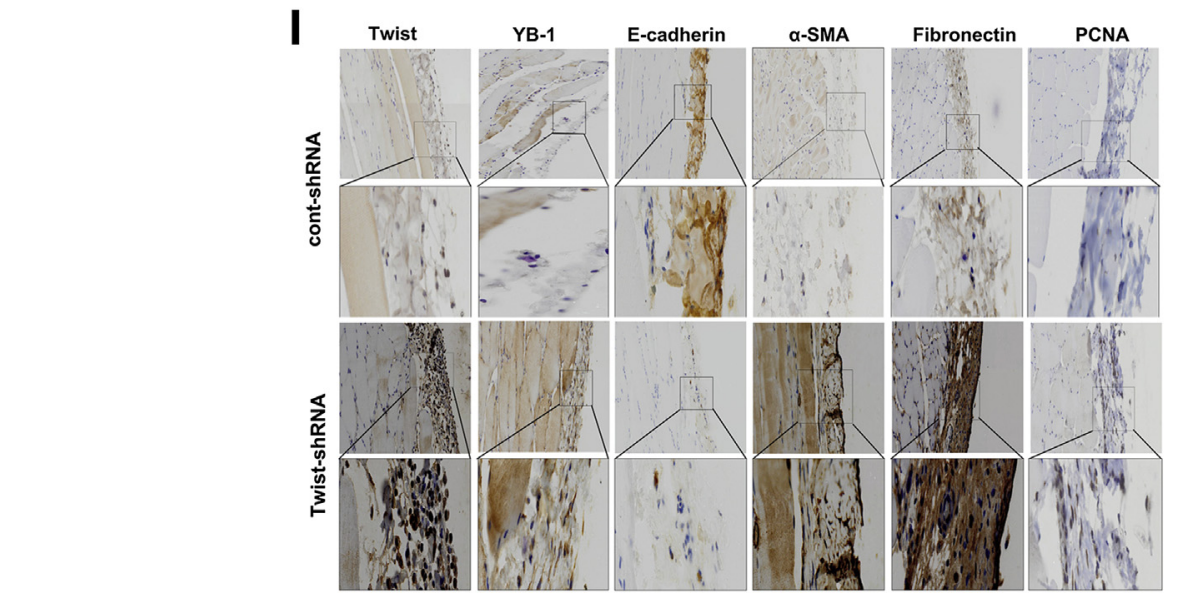

Figure 4 The effect of Twist knockdown by Twist-siRNA on the ability of human peritoneal mesothelial cells (HPMCs) to undergo epithelial-mesenchymal transition-induced fibrosis, growth, and cell cycle progression in vitro. A: Western blot analysis of Twist, YB-1, pYB-1, E-cadherin, and $\alpha$-smooth muscle actin $(\alpha-S M A)$ in HPMCs transfected with Twist-siRNA (siTwist) or control vector (cont), compared with high glucose (HG)-induced HPMCs (HG-HPMCs). B: Fluorescence microscopy shows the location and expression of Twist, YB-1, E-cadherin, and $\alpha$-SMA in HG-induced Twist-siRNA-treated HPMCs (siTwist) and control cells (HG-HPMCs cont). C: Real-time PCR shows the mRNA levels of Twist, YB-1, E-cadherin, and $\alpha$-SMA in HPMCs transfected with Twist-siRNA (siTwist), cont, and HG-induced HPMCs for 48 hours (HG-HPMCs). Bars represent the fold induction over untreated cells. D: The MTT assay shows the effect of Twist in the regulation of proliferation of HPMCs that were transfected with Twist-siRNA (siTwist), compared with cont in HG-induced HPMCs (HG-HPMCs cont). The monolayer growth rates of cells were determined by MTT assays. E: The histogram shows the average of cell cycle analysis in HG-induced HPMCs transfected with Twist-siRNA (siTwist), compared with the cont. Each experiment was conducted in triplicate. $\mathbf{F}$ and $\mathbf{G}$ : Cell cycle analysis shows that the inductions of $\mathrm{G}_{1}$ phase of HG-HPMCs transfected with cont (HG-HPMCs cont) and HG-HPMCs transfected with Twist-siRNA (siTwist) are $55.24 \%(\mathbf{F})$ and $83.5 \%(\mathbf{G})$, respectively. H: The histogram shows the thickness for hematoxylin and eosin staining in the submesothelial compact zone in Twist shRNA HG-PDF rats (61.50 \pm 23.57 ) compared with cont-shRNA HG-PDF rats $(121.50 \pm 14.34)$. I: Staining for the Twist, YB-1, fibronectin, E-cadherin, $\alpha$-SMA, and proliferating cell nuclear antigen (PCNA) in peritoneal samples by immunohistochemistry in Twist shRNA HG - peritoneal dialysis fluid (PDF) rats compared with cont-shRNA HG-PDF rats in vivo. Data are given as means \pm SEM (C, $\mathbf{D}$, and $\mathbf{H}) . n=3$ independent experiments conducted in duplicate $(\mathbf{C}) ; n=3$ or more separate experiments $(\mathbf{D})$; $n=8\left(\right.$ H). ${ }^{*} P<0.05$. Original magnifications: $\times 200($ B) $; \times 100$ (I, top panels); $\times 400$ (I, bottom panels).

fibrosis and cell proliferation, decreasing cell cycle arrest, and preventing HG-induced alterations.

\section{The Down-Regulation of YB-1 Decreases HG-Induced Fibrosis of HPMCs and Inhibits Cell Proliferation and Cell Cycle Progress by the Down-Regulation of Cyclin $D$ and CDK}

To obtain further insight into the biological relevance of YB-1, we analyzed its effect on fibrosis, proliferation, cell cycle progression, and changes in the expression of cell cycle proteins in immortal HPMCs after HG stimulation. Western blot analysis showed that YB-1 and $\alpha$-SMA protein levels significantly decreased and that E-cadherin protein levels significantly increased after transfection of siRNA against YB-1 (Figure 6A). MMP-2, which was overexpressed after HG stimulation, also significantly decreased in HG-HPMCs after transfection with YB-1-shRNA, compared with control (transfected with control-shRNA) (Supplemental Figure S3). But MMP-9 expression did not change after HG stimulation and transfection with YB-1-shRNA (Supplemental Figure S3). The results of 

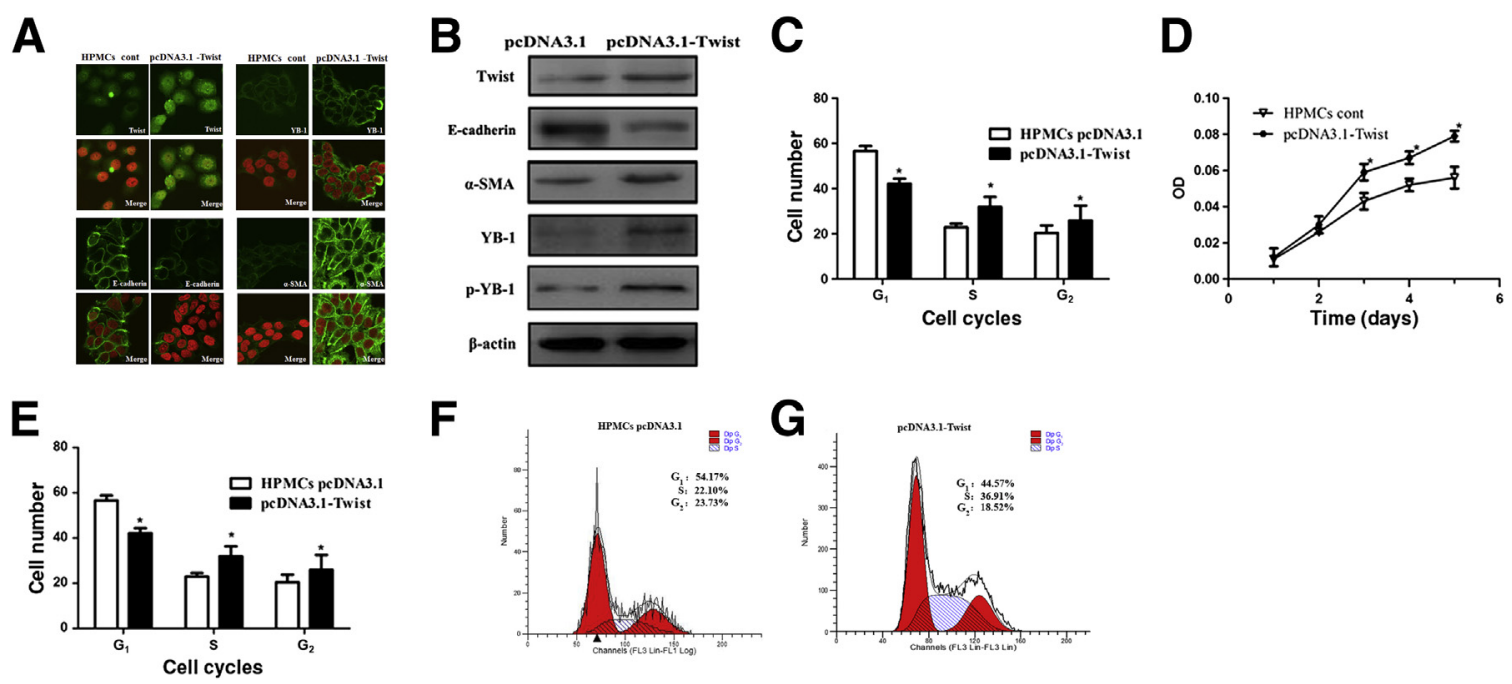

Figure 5 Up-regulation of Twist by a Twist plasmid affects the epithelial-mesenchymal transition-induced fibrosis, proliferation, and cell cycle arrest in human peritoneal mesothelial cells (HPMCs) in vitro. A: Fluorescence microscopy illustrates the location and expression of the Twist, YB-1, E-cadherin, and $\alpha$-smooth muscle actin ( $\alpha$-SMA) proteins in HPMCs transfected with a Twist plasmid (pcDNA3.1-Twist) or a control plasmid (HPMC pcDNA3.1). B: Western blot analysis of Twist, YB-1, pYB-1, E-cadherin, and $\alpha$-SMA in HPMCs transfected with a Twist plasmid (pcDNA3.1-Twist) or a control plasmid (HPMC pcDNA3.1). C: Real-time PCR shows the mRNA levels of Twist, YB-1, E-cadherin, and $\alpha$-SMA in HPMCs transfected with a Twist plasmid (pcDNA3.1-Twist) or a control plasmid (HPMC pcDNA3.1). D: Effect of Twist in the regulation of proliferation of HPMCs transfected with a Twist plasmid (pcDNA3.1-Twist) or a control plasmid (HPMC pcDNA3.1). Monolayer growth rates of cells were determined by MTT assays. E: The histogram shows the average of cell cycle analysis in HPMCs transfected with a Twist plasmid (pcDNA3.1-Twist) compared with a control plasmid (HPMC pcDNA3.1). Each experiment was conducted in triplicate. $\mathbf{F}$ and G: Cell cycle analysis in HPMCs transfected with pcDNA3.1-Twist or a control plasmid (pcDNA3.1) shows the proliferation induction of HPMCs that were transfected with control plasmid (HPMCs pcDNA3.1) or Twist plasmid (pcDNA3.1-Twist) as 54.17\% (F) and 44.57\% (G), respectively. Representative experiments of three were performed, with similar results. Data are given as means \pm SEM (D). $n=3$ or more separate experiments (D). $* P<0.05$. Original magnification, $\times 200(A)$.

the real-time PCR experiment and immunohistochemistry were similar to those of the Western blot analysis (Figure 6, $\mathrm{B}$ and $\mathrm{C})$. These data show that the down-regulation of YB-1 can inhibit EMT-induced fibrosis. The results of the MTT assays illustrated that the growth arrest was induced by YB-1-specific siRNA in HPMCs compared with the control HG-induced cells (Figure 6D). The downregulation of YB-1 expression led to a significant and reproducible increase in the proportion of HPMCs in the $\mathrm{G}_{1}$ phase (from $47.25 \% \pm 2.75 \%$ to $80.93 \% \pm 2.98 \%$ ), and PI staining decreased from $52.75 \%$ to $19.09 \%$ (Figure 6, $\mathrm{E}-\mathrm{G})$. To explore the underlying molecular mechanism of YB-1-induced cell cycle arrest, we detected the expression of cell cycle-related molecules after transfection (Figure 6H and Supplemental Figure S4). Our results indicated that the down-regulation of YB-1 protein expression was associated with a reduction in cyclin D1 and CDK4 and cyclin $\mathrm{E}$ and CDK2 expression, but was associated with an increase in p27Kip1 expression. Therefore, we infer that the downregulation of YB-1 might also result in a decrease in cellular proliferation, $\mathrm{G}_{1} / \mathrm{S}$ cell cycle arrest, and enhanced fibrosis after changes in various cell cycle proteins.

We further established PD animal model treatment with YB-1-shRNA lentivirus compared with control-shRNA lentivirus (Figure 6, I and J). A thinner PM was found as $69.00 \pm 25.38 \mu \mathrm{m}$ in the parietal peritoneum in YB1-shRNA PD rats compared with the control shRNA group (Figure 6J). An analysis of Twist, YB-1,
E-cadherin, $\alpha$-SMA, FN, and PCNA by immunohistochemistry indicated that injection of YB-1-shRNA in PD rats caused down-regulation of YB-1, $\alpha$-SMA, FN, and PCNA, but up-regulation of E-cadherin in the PM. The expression of Twist did not change after YB-1 down-regulation. The down-regulation of FN, PCNA, and EMT marker $\alpha$ SMA showed that down-regulation of YB-1 caused alleviation of PM fibrosis and a slower peritoneal transport rate for lower molecular weight (Supplemental Figure S2) and growth retardation of HPMCs in PD rats.

\section{Discussion}

The peritoneal mesothelium plays a pivotal role in the control of peritoneal homeostasis. In a series of experiments, the loss of the mesothelium over time and the disruption of the interstitium after chronic exposure to glucose-containing PDF were demonstrated. ${ }^{26,27}$ Previous studies have also shown that exposure to HG could damage HPMCs both in vivo and in vitro, ${ }^{28,29}$ and it was considered to be the primary cause of peritoneal fibrosis. The inhibitory effect of HG concentrations on the proliferation of HPMCs is not solely related to some property of glucose itself, but rather it is related to its metabolic effects. ${ }^{30,31}$

We sought to use HG to reproduce the damage caused by the PD effluent and the profibrotic stimuli that are induced during PM fibrosis. HG can induce EMT in HPMCs, and 

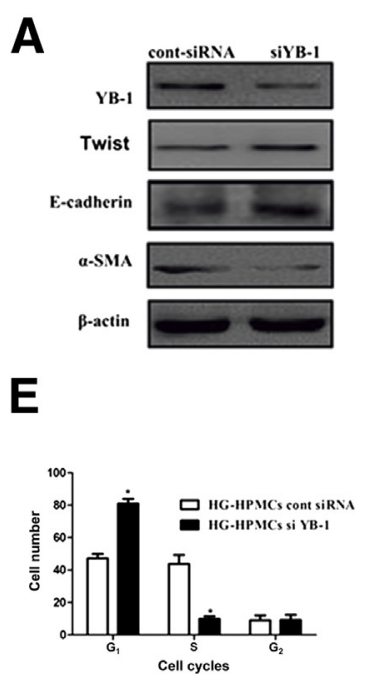

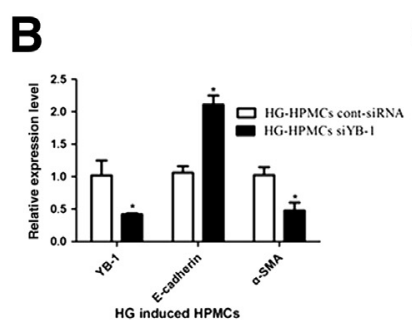

C

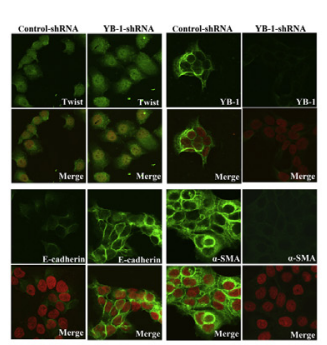

$\mathbf{F}$
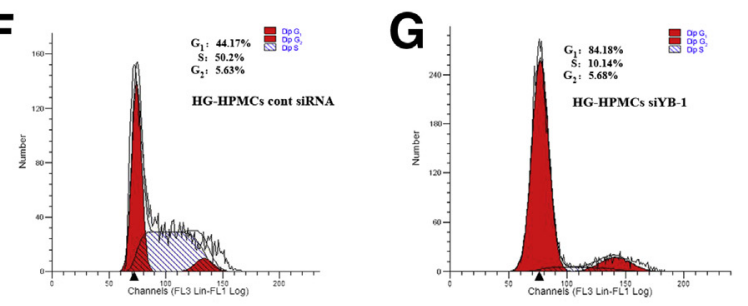

I

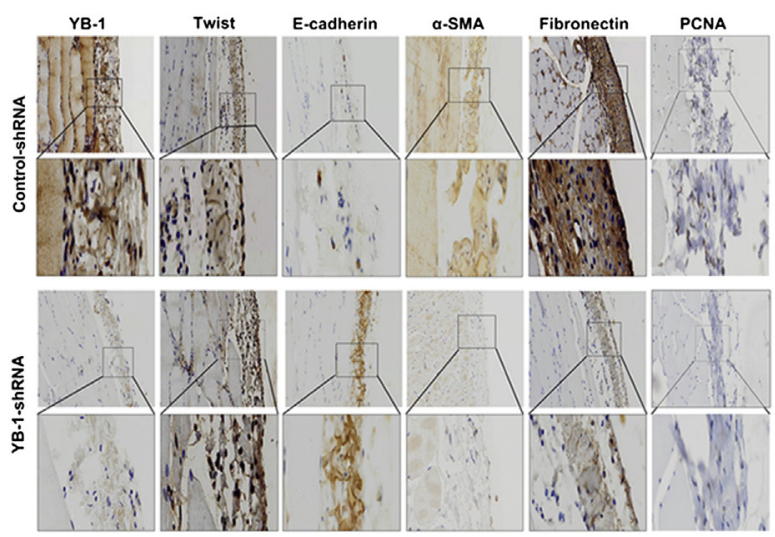

D
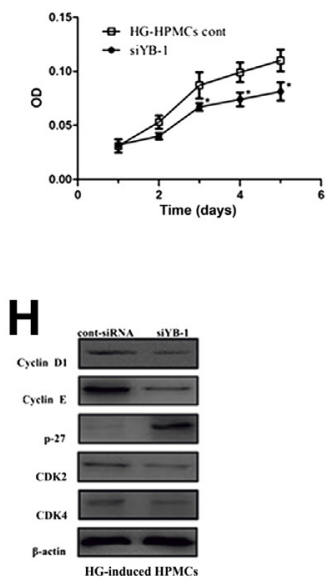

$\mathbf{J}$

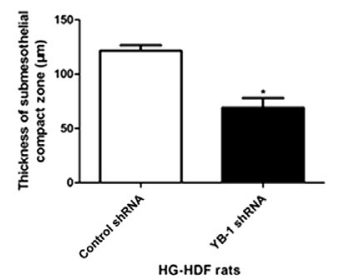

Figure 6 The knockdown of YB-1 by siRNA affected the ability of human peritoneal mesothelial cells (HPMCs) to undergo epithelial-mesenchymal transition-induced fibrosis, growth, and cell cycle progression in vitro. A: Western blot analysis of Twist, YB-1, E-cadherin, and $\alpha$-smooth muscle actin ( $\alpha$-SMA) in HPMCs transfected with YB-1-siRNA (siYB-1) compared with control vector (cont) in high glucose (HG)-induced HPMCs (HG-HPMCs). B: Real-time PCR shows the mRNA levels of YB-1, E-cadherin, and $\alpha$-SMA in HPMCs transfected with siYB-1 and cont in HG-induced HPMCs for 48 hours (HG-HPMCs). Bars represent the fold induction over untreated cells. C: Fluorescence microscopy shows the location and expression of Twist, YB-1, E-cadherin, and $\alpha$-SMA in HG-HPMCs transfected with YB-1-shRNA lentivirus compared with transfected control-shRNA cells. D: The effect of YB-1 in the regulation of the proliferation of HPMCs transfected with siYB-1, compared with cont in HG-induced HPMCs. Monolayer growth rates of the cells were determined by MTT assays. E: The histogram shows the average of cell cycle analysis in HG-HPMCs transfected with siYB-1, compared with cont. Each experiment was conducted in triplicate. F and G: Cell cycle analysis in HG-induced HPMC cells transfected with siYB-1 or a cont shows the proliferation induction of HPMCs that were transfected with siYB-1 vector as $84.18 \%$ (F) compared with transfected control plasmid for $44.17 \%$ (G), respectively. Representative experiment of three with similar results. H: The effect of YB-1 on the expression of cell cycle effectors by Western blot analysis. All examined gene expression levels were quantitatively analyzed and are expressed as ratios over $\beta$-actin. This is a representative experiment of three separate experiments with similar results. The expression of cyclin D1, cyclin E, cyclin-dependent kinase (CDK) 2, CDK4, and p27 proteins was evaluated after infection with cont siRNA or siYB-1 in HG-induced HPMCs. I: Staining for Twist, YB-1, E-cadherin, $\alpha$-SMA, fibronectin, and proliferating cell nuclear antigen (PCNA) in peritoneal membrane by immunohistochemistry in YB-1-shRNA HG-peritoneal dialysis fluid (PDF) rats compared with cont-shRNA HG-PDF rats in vivo. J: The histogram shows the thickness for hematoxylin and eosin staining in the submesothelial compact zone in YB-1 shRNA HG-PDF rats $(69.00 \pm 25.38)$ compared with cont-shRNA HG-PDF rats (121.50 \pm 14.34$)$. Data are given as means \pm SEM (B, D, and J). $n=3$ independent experiments conducted in duplicate (B); $n=3$ or more separate experiments $(\mathbf{D})$. ${ }^{*} P<0.05 .0$ riginal magnifications: $\times 200($ C); $\times 100$ (I, top panels); $\times 400$ (I, bottom panels).

HG exerts additional effects on cell morphology, which finally leads to PM fibrosis. ${ }^{32}$ It was thought that soon after dialysis is initiated, peritoneal mesothelial cells undergo a transition from an epithelial phenotype of cobblestone-like morphology to a mesenchymal phenotype of fibroblastlike morphology. Some studies have shown that even much early in CAPD, a loss of cuboid morphology and expression of E-cadherin are observed both in vivo and ex vivo, even when cells retain an epithelioid appearance. Continued PD results in long-term exposure to mechanical denudation. ${ }^{20}$ In vitro data suggested that EMT is induced after HG stimulation for 2 days, and could be reversed after changing the HG cell culture fluid to the normal glucose culture medium in $<7$ days. However, EMT reversion does not happen after HG stimulation for $>7$ days. So, HGstimulated EMT of HPMC can be reversed at an early time stage but becomes irreversible at a later stage. ${ }^{5,16}$ A time dependence of EMT reversal was also found when using rapamycin, to induce EMT of mesothelial cells at early time points, but was unable to inhibit the fibroblast-like 
phenotypic transformation on day 7 of transforming growth factor- $\beta$ stimulation. Moreover, HG-induced phenotypic transition was observed as early as 48 hours of stimulation before any significant morphological changes of cells, which suggests that HG-induced alteration in cell phenotype may be one of the earliest phenomena of peritoneal damage. Although peritoneal fibrosis was regarded as a relentless process once it started, HG stimulation for 48 hours, followed by a removal, resulted in a significant reversal of EMT of HPMCs with epithelial redifferentiation to prestimulation level.

Some studies have shown that $\mathrm{HG}$ can alter the proliferation of HPMCs, following the changes in the cell cycle. ${ }^{33} \mathrm{We}$ observed that cell proliferation is not affected, or even inhibited, at the initial stimulation time for 48 hours; proliferation increases and cell cycle progresses rapidly after several days of HG stimulation. It is possible that at the initial HG stimulation time, cells encounter stress damage because of $\mathrm{HG}$, and later HPMCs are rescued but show abnormal proliferation.

Transcription factors, both Twist and YB-1, were protooncogenes and are known to regulate the invasive and metastatic characteristics of cancer cells. ${ }^{7,34,35}$ In bladder cancer, Twist regulates YB-1 and is involved in cell growth, invasion, motility, and resistance to cisplatin and doxorubicin. ${ }^{36}$ Twist also regulates EMT and is involved in tumor metastasis through YB-1. Inhibition of Twist can decrease the expression of YB-1 in highly metastatic cells and finally suppresses the metastatic ability of tumor cells. ${ }^{7}$

Twist induces EMT by HG stimulation of HPMCs in vitro, and might even promote PM fibrosis, according to our previous studies. ${ }^{11}$ Our earlier study was only an initial exploration into the role of Twist in PM fibrosis, which contributed data about the molecular mechanism of Twist in vitro, but not in vivo. Thus, we continued to study the effects of Twist in vivo to identify its possible targets in PM fibrosis. Our data showed that Twist and YB-1 positively correlated and that both were upregulated in deciduous HPMCs from the effluents of patients who underwent long-term PD.

YB-1 is expressed in cells and is the core EMT regulator that participated in tumor metastasis and growth. ${ }^{13}$ YB-1 promotes cell proliferation through the transactivation of target genes, such as proliferating cell nuclear antigen. ${ }^{37}$ On the basis of these reports, we proposed that Twist might regulate the expression of YB-1 and might be involved in the promotion of cell proliferation and EMT-induced migration. ${ }^{7,15,38}$ Structural analysis of the YB-1 promoter reveals that several E-boxes may participate in the regulation of YB-1 expression. YB-1-null mice and Twist-null embryos both exhibit failure of neural tube closure, ${ }^{15,38,39}$ which suggests that YB-1 might be a major target of Twist. YB-1 inhibits the translation of the mRNAs encoding $\mathrm{CDH} 1$ by snail and other factors, which often appears to involve EMT and contributes to motile tumor cells. YB-1 appears to promote tumor activities by controlling cyclin D1, CDK2, cyclin E, and CDK4; both these processes promote cell cycle progression. YB-1 protein, as a RNA binding protein, can bind to the promoter of $P C N A$, and controls PCNA expression in non-small cell lung cancer cells. ${ }^{40}$ So, the Twist-YB-1 pathway likely plays an active role in tumor cell EMT and motility, proliferation and growth, cell cycle progression, and malignancy.

Twist overexpression in gastric cancer cells promotes the expression of cyclin D1 and MMP-2, whereas its suppression reduces cyclin D1 expression and MMP-2 activity. YB-1 transcriptionally regulates several MMPs, including MMP-2. Whether YB-1 activates or represses MMPs depends on the cellular context. MMP-2 and MMP-9 are fundamental enzymes in extracellular matrix homeostasis. MMP-2 is specifically implicated in EMT, and inhibition of MMP-2 prevents EMT in renal tubular cell culture. ${ }^{40-42}$ We also found that MMP-2, but not MMP-9, was overexpressed in HG-HPMCs compared with normal HPMCs. We also showed a significant decrease in the expression of MMP-2, but not MMP-9, after transfection with YB-1 shRNA in HG-HPMCs (Supplemental Figure S3).

We conclude that the subcellular localization of Twist and YB-1 might affect the changes in the cellular morphology and behavior of HPMCs, which might be associated with altered signaling pathways, subsequent growth, cell cycle changes, and fibrosis. The silencing of both Twist and YB-1 induced $\mathrm{G}_{1} / \mathrm{S}$ arrest, and decelerated the growth of HPMCs. In addition, the decrease in growth of HPMCs might be partially regulated by various cell cycle proteins, such as cyclin and CDK complexes that function in the control of $G_{1}$ to $S$ progression. Furthermore, our study also suggested that YB-1 is a major downstream target gene of Twist in HPMCs. This functional link between YB-1 and Twist suggested that Twist and its downstream effector, YB-1, might serve as promising molecular targets for the treatment of peritoneal fibrosis. Additional knockout animal models of chronic PD might provide more answers. This study is the first extensive characterization of a signaling pathway that controls cell proliferation and EMT-induced fibrosis in a nontumor primary cell culture model and provides basic knowledge and clinical relevance. These results could form the rationale for the development of drugs that can counteract the progressive deterioration of the PM that occurs in patients with CAPD.

\section{Acknowledgments}

We thank Yongqiang Ding (Laideer Biology Company, Guangzhou, Guangdong, China) for suggestions and technical support with the chromatin immunoprecipitation assay and the reporter assay and Prof. Carlotta A. Glackin (National Medical Center and Beckman Research Institute, Durate, CA) for the pcDNA3.1-Twist plasmid.

L.H. conceived and designed the experiments; M.C., J.H., S.L., Z.J., and W.L. performed the experiments; L.H. and H.W. analyzed the data; J.Y., C.L., S.S., H.W., X.C., and L.H. contributed reagents, materials, and analysis tools; and L.H. wrote the manuscript. 


\section{Supplemental Data}

Supplemental material for this article can be found at http://dx.doi.org/10.1016/j.ajpath.2015.04.008.

\section{References}

1. Jain AK, Blake P, Cordy P, Garg AX: Global trends in rates of peritoneal dialysis. J Am Soc Nephrol 2012, 23:533-544

2. Williams JD, Craig KJ, Topley N, Von Ruhland C, Fallon M, Newman GR, Mackenzie RK, Williams GT: Morphologic changes in the peritoneal membrane of patients with renal disease. J Am Soc Nephrol 2002, 13:470-479

3. Aroeira LS, Aguilera A, Sanchez-Tomero JA, Bajo MA, Del PG, Jimenez-Heffernan JA, Selgas R, Lopez-Cabrera M: Epithelial to mesenchymal transition and peritoneal membrane failure in peritoneal dialysis patients: pathologic significance and potential therapeutic interventions. J Am Soc Nephrol 2007, 18:2004-2013

4. Lamouille S, Subramanyam D, Blelloch R, Derynck R: Regulation of epithelial-mesenchymal and mesenchymal-epithelial transitions by microRNAs. Curr Opin Cell Biol 2013, 25:200-207

5. Yu MA, Shin KS, Kim JH, Kim YI, Chung SS, Park SH, Kim YL, Kang DH: HGF and BMP-7 ameliorate high glucose-induced epithelial-to-mesenchymal transition of peritoneal mesothelium. J Am Soc Nephrol 2009, 20:567-581

6. Yung S, Chen XR, Tsang RC, Zhang Q, Chan TM: Reduction of perlecan synthesis and induction of TGF-beta1 in human peritoneal mesothelial cells due to high dialysate glucose concentration: implication in peritoneal dialysis. J Am Soc Nephrol 2004, 15: $1178-1188$

7. Yang J, Mani SA, Donaher JL, Ramaswamy S, Itzykson RA, Come C, Savagner P, Gitelman I, Richardson A, Weinberg RA: Twist, a master regulator of morphogenesis, plays an essential role in tumor metastasis. Cell 2004, 117:927-939

8. Yang J, Mani SA, Weinberg RA: Exploring a new twist on tumor metastasis. Cancer Res 2006, 66:4549-4552

9. Pozharskaya V, Torres-Gonzalez E, Rojas M, Gal A, Amin M, Dollard S, Roman J, Stecenko AA, Mora AL: Twist: a regulator of epithelial-mesenchymal transition in lung fibrosis. PLoS One 2009, 4: e7559

10. Sun S, Ning X, Zhang Y, Lu Y, Nie Y, Han S, Liu L, Du R, Xia L, He L, Fan D: Hypoxia-inducible factor-1alpha induces Twist expression in tubular epithelial cells subjected to hypoxia, leading to epithelial-to-mesenchymal transition. Kidney Int 2009, $75: 1278-1287$

11. Li C, Ren Y, Jia X, Liang P, Lou W, He L, Li M, Sun S, Wang H: Twist overexpression promoted epithelial-to-mesenchymal transition of human peritoneal mesothelial cells under high glucose. Nephrol Dial Transplant 2012, 27:4119-4124

12. Vesuna F, van Diest P, Chen JH, Raman V: Twist is a transcriptional repressor of E-cadherin gene expression in breast cancer. Biochem Biophys Res Commun 2008, 367:235-241

13. Kuwano M, Oda Y, Izumi H, Yang SJ, Uchiumi T, Iwamoto Y, Toi M, Fujii T, Yamana H, Kinoshita H, Kamura T, Tsuneyoshi M, Yasumoto K, Kohno K: The role of nuclear Y-box binding protein 1 as a global marker in drug resistance. Mol Cancer Ther 2004, 3: 1485-1492

14. Basaki Y, Hosoi F, Oda Y, Fotovati A, Maruyama Y, Oie S, Ono M, Izumi H, Kohno K, Sakai K, Shimoyama T, Nishio K, Kuwano M: Akt-dependent nuclear localization of Y-box-binding protein 1 in acquisition of malignant characteristics by human ovarian cancer cells. Oncogene 2007, 26:2736-2746

15. Shiota M, Izumi H, Onitsuka T, Miyamoto N, Kashiwagi E, Kidani A, Yokomizo A, Naito S, Kohno K: Twist promotes tumor cell growth through YB-1 expression. Cancer Res 2008, 68:98-105
16. He L, Lou W, Ji L, Liang W, Zhou M, Xu G, Zhao L, Huang C, Li R, Wang H, Chen X, Sun S: Serum response factor accelerates the high glucose-induced epithelial-to-mesenchymal transition (EMT) via snail signaling in human peritoneal mesothelial cells. PLoS One 2014, 9: e108593

17. Committee for the Update of the Guide for the Care and Use of Laboratory Animals: National Research Council: Guide for the Care and Use of Laboratory Animals: Eighth Edition. Washington, DC, National Academies Press, 2011

18. Stylianou E, Jenner LA, Davies M, Coles GA, Williams JD: Isolation, culture and characterization of human peritoneal mesothelial cells. Kidney Int 1990, 37:1563-1570

19. Lopez-Cabrera M, Aguilera A, Aroeira LS, Ramirez-Huesca M, Perez-Lozano ML, Jimenez-Heffernan JA, Bajo MA, Del PG, Sanchez-Tomero JA, Selgas R: Ex vivo analysis of dialysis effluentderived mesothelial cells as an approach to unveiling the mechanism of peritoneal membrane failure. Perit Dial Int 2006, 26:26-34

20. Yanez-Mo M, Lara-Pezzi E, Selgas R, Ramirez-Huesca M, Dominguez-Jimenez C, Jimenez-Heffernan JA, Aguilera A, SanchezTomero JA, Bajo MA, Alvarez V, Castro MA, Del PG, Cirujeda A, Gamallo C, Sanchez-Madrid F, Lopez-Cabrera M: Peritoneal dialysis and epithelial-to-mesenchymal transition of mesothelial cells. N Engl J Med 2003, 348:403-413

21. Bidmon B, Endemann M, Arbeiter K, Ruffingshofer D, Regele H, Herkner K, Eickelberg O, Aufricht C: Overexpression of HSP-72 confers cytoprotection in experimental peritoneal dialysis. Kidney Int 2004, 66:2300-2307

22. Aroeira LS, Aguilera A, Selgas R, Ramirez-Huesca M, PerezLozano ML, Cirugeda A, Bajo MA, Del PG, Sanchez-Tomero JA, Jimenez-Heffernan JA, Lopez-Cabrera M: Mesenchymal conversion of mesothelial cells as a mechanism responsible for high solute transport rate in peritoneal dialysis: role of vascular endothelial growth factor. Am J Kidney Dis 2005, 46:938-948

23. Lee SH, Kang HY, Kim KS, Nam BY, Paeng J, Kim S, Li JJ, Park JT, Kim DK, Han SH, Yoo TH, Kang SW: The monocyte chemoattractant protein-1 (MCP-1)/CCR2 system is involved in peritoneal dialysisrelated epithelial-mesenchymal transition of peritoneal mesothelial cells. Lab Invest 2012, 92:1698-1711

24. He L, Wang H, Jin H, Guo C, Xie H, Yan K, Li X, Shen Q, Qiao T, Chen G, Chai N, Zhao L, Dong Q, Zheng Y, Liu J, Fan D: CIAPIN1 inhibits the growth and proliferation of clear cell renal cell carcinoma. Cancer Lett 2009, 276:88-94

25. Arva NC, Gopen TR, Talbott KE, Campbell LE, Chicas A, White DE, Bond GL, Levine AJ, Bargonetti J: A chromatin-associated and transcriptionally inactive p53-Mdm2 complex occurs in mdm2 SNP309 homozygous cells. J Biol Chem 2005, 280:26776-26787

26. Gotloib L, Shostak A, Wajsbrot V, Kushnier R: High glucose induces a hypertrophic, senescent mesothelial cell phenotype after long in vivo exposure. Nephron 1999, 82:164-173

27. Ksiazek K, Korybalska K, Jorres A, Witowski J: Accelerated senescence of human peritoneal mesothelial cells exposed to high glucose: the role of TGF-beta1. Lab Invest 2007, 87:345-356

28. Jorres A, Gahl GM, Frei U: Peritoneal dialysis fluid biocompatibility: does it really matter? Kidney Int Suppl 1994, 48:S79-S86

29. Shao JC, Yorioka N, Nishida Y, Yamakido M: Effect of $\mathrm{pH}$ and glucose on cultured human peritoneal mesothelial cells. Scand J Urol Nephrol 1999, 33:248-256

30. Ito T, Yorioka N, Kyuden Y, Asakimori Y, Kiribayashi K, Ogawa T, Kohno N: Effect of glucose polymer on the intercellular junctions of cultured human peritoneal mesothelial cells. Nephron Clin Pract 2003, 93:c97-c105

31. Ito T, Yorioka N, Yamamoto M, Kataoka K, Yamakido M: Effect of glucose on intercellular junctions of cultured human peritoneal mesothelial cells. J Am Soc Nephrol 2000, 11:1969-1979

32. Iwano M, Plieth D, Danoff TM, Xue C, Okada H, Neilson EG: Evidence that fibroblasts derive from epithelium during tissue fibrosis. J Clin Invest 2002, 110:341-350 
33. Chan TM, Leung JK, Sun Y, Lai KN, Tsang RC, Yung S: Different effects of amino acid-based and glucose-based dialysate from peritoneal dialysis patients on mesothelial cell ultrastructure and function. Nephrol Dial Transplant 2003, 18:1086-1094

34. Cheng GZ, Chan J, Wang Q, Zhang W, Sun CD, Wang LH: Twist transcriptionally up-regulates AKT2 in breast cancer cells leading to increased migration, invasion, and resistance to paclitaxel. Cancer Res 2007, 67:1979-1987

35. Evdokimova V, Ruzanov P, Anglesio MS, Sorokin AV, Ovchinnikov LP, Buckley J, Triche TJ, Sonenberg N, Sorensen PH: Akt-mediated YB-1 phosphorylation activates translation of silent mRNA species. Mol Cell Biol 2006, 26:277-292

36. Shiota M, Yokomizo A, Itsumi M, Uchiumi T, Tada Y, Song Y, Kashiwagi E, Masubuchi D, Naito S: Twistl and Y-box-binding protein-1 promote malignant potential in bladder cancer cells. BJU Int 2011, 108:E142-E149

37. Kohno K, Uchiumi T, Niina I, Wakasugi T, Igarashi T, Momii Y, Yoshida T, Matsuo K, Miyamoto N, Izumi H: Transcription factors and drug resistance. Eur J Cancer 2005, 41:2577-2586
38. Stratford AL, Fry CJ, Desilets C, Davies AH, Cho YY, Li Y, Dong Z, Berquin IM, Roux PP, Dunn SE: Y-box binding protein-1 serine 102 is a downstream target of $\mathrm{p} 90$ ribosomal S6 kinase in basal-like breast cancer cells. Breast Cancer Res 2008, 10:R99

39. Soo K, O'Rourke MP, Khoo PL, Steiner KA, Wong N, Behringer RR, Tam PP: Twist function is required for the morphogenesis of the cephalic neural tube and the differentiation of the cranial neural crest cells in the mouse embryo. Dev Biol 2002, 247:251-270

40. Yoshimatsu T, Uramoto H, Oyama T, Yashima Y, Gu C, Morita M, Sugio K, Kohno K, Yasumoto K: Y-box-binding protein-1 expression is not correlated with $\mathrm{p} 53$ expression but with proliferating cell nuclear antigen expression in non-small cell lung cancer. Anticancer Res 2005, 25:3437-3443

41. Devuyst O, Margetts PJ, Topley N: The pathophysiology of the peritoneal membrane. J Am Soc Nephrol 2010, 21:1077-1085

42. Lasham A, Print CG, Woolley AG, Dunn SE, Braithwaite AW: YB-1: oncoprotein, prognostic marker and therapeutic target? Biochem J 2013, 449:11-23 